\title{
ESTRATÉGIAS PARA O ENVOLVIMENTO DA FAMÍLIA EM UM CUIDADO SEGURO
}

\author{
STRATEGIES FOR FAMILY INVOLVEMENT IN SAFE CARE
}

ESTRATEGIAS PARA LA PARTICIPACIÓN FAMILIAR EN EL CUIDADO SEGURO

\begin{abstract}
Cecília Siqueira ${ }^{1}$ Aline de Pinho Dias $^{2}$

\section{Resumo}

Com o objetivo de levantar as estratégias utilizadas pelas instituições de saúde para envolver o paciente e sua família no processo seguro do cuidado, foi realizada uma revisão da literatura, mediante artigos encontrados nas bases LILAC, SCIELO, PUB-MED e CAPES, utilizando-se dos descritores: estratégias de segurança do paciente, comunicação, identificação do paciente, higienização das mãos e quedas. Foram selecionados artigos publicados no período compreendido entre 2012 e dezembro de 2019. Pelos critérios de exclusão/inclusão foram analisados 18 artigos relacionados ao objetivo proposto. As estratégias propostas pela revisão de literatura forneceram a dimensão de novas perspectivas no tocante ao cuidado seguro do paciente. A partir dos artigos estudados, conclui-se que ainda é incipiente a divulgação sobre o cuidado seguro aos pacientes e, portanto, deve haver continuidade nos estudos sobre o tema, além da adoção de estratégias capazes de validar a segurança do paciente.
\end{abstract}

Palavras-chave: Segurança do Paciente. Comunicação. Envolvimento.

\begin{abstract}
In order to raise the strategies used by health institutions to involve patients and their families in the safe care process, a literature review was carried out, using articles found in LILAC, SCIELO, PUB-MED and CAPES databases, using of the descriptors: patient safety strategies, communication, patient identification, hand hygiene and falls. Articles published in the period between 2012 and December 2019 were selected. Through the exclusion / inclusion criteria, 18 articles related to the proposed objective were analyzed. The strategies proposed by the literature review provided the dimension of new perspectives regarding safe patient care. From the articles studied, it is concluded that the dissemination of safe care for patients is still incipient and, therefore, there must be continuity in studies on the subject, in addition to the adoption of strategies capable of validating patient safety.
\end{abstract}

Keywords: Patient safety. Communication. Involvement

\section{Resumen}

Con el fin de plantear las estrategias que utilizan las instituciones de salud para involucrar a los pacientes y sus familias en el proceso de atención segura, se realizó una revisión de la literatura, utilizando artículos encontrados en las bases de datos LILAC, SCIELO, PUB-MED y CAPES, utilizando de los descriptores: estrategias de seguridad del paciente, comunicación, identificación del paciente, higiene de manos y caídas. Se seleccionaron los artículos publicados en el período comprendido entre 2012 y diciembre de 2019. Mediante los criterios de exclusión / inclusión se analizaron 18 artículos relacionados con el objetivo propuesto. Las estrategias propuestas por la revisión de la literatura aportaron la dimensión de nuevas perspectivas sobre la atención segura al paciente. De los artículos estudiados se concluye que la difusión de la atención segura al paciente es aún incipiente y, por tanto, debe haber continuidad en los estudios sobre el tema, además de la adopción de estrategias capaces de validar la seguridad del paciente.

Palabras clave: Seguridad del paciente. Comunicación. Envolvimiento

\footnotetext{
${ }^{1}$ Mestranda em Gestão e Inovação em Saúde pela Universidade Federal do Rio Grande do Norte. Analista administrativo/gestão hospitalar na Empresa Brasileira de Serviços Hospitalares. E-mail: ceciliasiq@gmail.com

2 Doutora em Educação pela UFRN. Departamento de Fundamentos e politicas da educação. Professora do Programa de Pós Graduação em Gestão e Inovação em Saúde.
} 


\section{INTRODUÇÃO}

A segurança do paciente tem sido fortemente debatida nos últimos anos, sobretudo por estar associada ao sinônimo de qualidade da assistência nos serviços de saúde. A Organização Mundial de Saúde (OMS) a define como a redução ao mínimo aceitável do risco de dano desnecessário associado ao cuidado de saúde (BRASIL, 2013).

A ocorrência dos eventos adversos (EA) afeta de $4 \%$ a $16 \%$ de pacientes hospitalizados em países desenvolvidos. Esse número pode ser ainda maior em países em desenvolvimento (BRASIL, 2017a). Por isso, essa temática representa um dos maiores desafios para os serviços de saúde, o que tem mobilizado o mundo na busca por estratégias que garantam uma assistência em saúde de qualidade e segura. O envolvimento do próprio paciente e dos cuidadores - que, na maioria das vezes, é um membro de sua família - nas ações de cuidado é uma das estratégias que tem sido estimulada para a prevenção desses incidentes.

Essa abordagem demanda mudanças de cultura nos serviços de saúde, de forma que os profissionais possam estimular e apoiar atitudes mais ativas dos usuários desses serviços. Assim, os pacientes devem ser considerados parceiros capazes de desempenhar um papel responsável por sua saúde e cuidado (BRASIL, 2017b).

No ano de 2004, a OMS criou a Aliança Mundial para Segurança do Paciente, com o objetivo principal de mobilizar esforços globais na segurança do cuidado em saúde para todos os pacientes (WHO, 2008). Em consonância, a Segurança do Paciente já faz parte da agenda política do Brasil e teve como ápice a publicação da portaria $n^{\circ} 529 / 2013$, que institui o Programa Nacional de Segurança do Paciente (PNSP).

É possível encontrar diversas iniciativas para a promoção da segurança e da qualidade na assistência à saúde em âmbito mundial. Entretanto, faz-se necessária maior sistematização da aplicação das melhores práticas em prol da segurança do paciente, de forma que elas sejam eficazes e não fiquem, somente, como projetos não aplicados, pois, senão, os esforços serão desperdiçados (MILAGRES, 2015).

A Organização Mundial de Saúde recomenda que uma das iniciativas para garantir a segurança do paciente seja o desenvolvimento da autonomia e corresponsabilidade do próprio paciente/acompanhante no processo de tratamento, recuperação e cura. Dessa forma, por meio do art. 3ํ da portaria ํㅗ 529/2013, o Ministério da Saúde estabelece como objetivos o envolvimento de pacientes e familiares nas ações de segurança do paciente, a ampliação do 
acesso da sociedade às informações relativas ao tema e à produção, sistematização e difusão de conhecimentos sobre segurança do paciente (BRASIL, 2013).

As orientações de segurança do paciente permanecem sendo desafios a serem incorporados à construção do cuidado seguro. É necessário que as famílias entendam a importância de sua participação ativa no cuidado ao paciente. Para isso, as equipes de saúde precisam se engajar no processo de educação e registro dessas orientações. É importante salientar, ainda, que todos os pacientes e acompanhantes devem ser orientados e estarem cientes das medidas de proteção para segurança do paciente durante a internação hospitalar. Para tal, definiu-se como questão norteadora: quais as estratégias utilizadas pelas instituições de saúde com foco no envolvimento dos pacientes e de sua família quanto aos requisitos apresentados pelas metas internacionais de segurança do paciente?

Nessa perspectiva, o presente estudo tem como objetivo levantar as estratégias utilizadas pelas instituições de saúde para envolver o paciente e sua família no processo de cuidar, por meio de revisão da literatura. A relevância dessa abordagem está em identificar, nos estudos mais recentes, evidências de ações que auxiliem o envolvimento do paciente e do acompanhante no cuidado. Por meio deste levantamento, possibilitam-se a reflexão e o debate sobre as estratégias encontradas, o que torna possível o aprimoramento da segurança do paciente de forma eficiente.

\section{METODOLOGIA}

Para atingir os objetivos propostos utilizou-se como metodologia a revisão bibliográfica qualitativa e descritiva. A revisão bibliográfica permite abarcar uma gama de fenômenos diferentemente dos que são pesquisados diretamente. A abordagem qualitativa, por sua vez, refere-se às percepções, crenças e opiniões produzidas pelos seres humanos. Finalmente, a metodologia descritiva permite descrever os eventos e as características de uma determinada população.

A pesquisa bibliográfica foi realizada nas bases de dados LILACS (Literatura Latino Americana e do Caribe em Ciências da Saúde), SCIELO (Scientific Electronic Library on line), PUBMED e CAPES (Coordenação de Aperfeiçoamento de Pessoal de Nível Superior). A escolha dessas bases deu-se em virtude da abrangência, gratuidade e integralidade dos artigos. Os descritores selecionados foram: estratégias de segurança do paciente, comunicação, identificação do paciente, higienização das mãos e quedas. 
Com a inserção dos descritores nas bases de dados, foram encontrados 244 artigos, delimitados pelo recorte temporal de 2012 a dezembro/2019, nas línguas espanhol, inglês e português. A partir desse levantamento inicial, realizou-se análise prévia do conteúdo dos textos, com base em leitura flutuante dos títulos e resumos de cada um deles, considerando a sua inserção nas temáticas da segurança do paciente e cuidado seguro, com foco no envolvimento do paciente, familiares e acompanhantes. Foram excluídos os artigos que tratavam da perspectiva dos profissionais, infraestrutura e outros assuntos que não envolvem o paciente/familiar nos processos de segurança.

Após essa primeira etapa foram selecionados 18 artigos que atendiam aos requisitos da pesquisa. Os resultados extraídos dos artigos foram organizados de acordo com o título, ano de publicação, objetivo, tipo de estudo, tipo de amostra e principal contribuição.

\section{RESULTADOS}

Os resultados provenientes da análise dos 18 artigos foram organizados no Quadro 1:

Quadro 1 - Apontamentos dos autores

\begin{tabular}{|c|c|c|c|}
\hline $\begin{array}{l}\text { Autor/Ano de } \\
\text { Publicação }\end{array}$ & $\begin{array}{l}\text { Título/Ano de } \\
\text { Publicação }\end{array}$ & Objetivo/Tipo de Estudo & $\begin{array}{c}\text { Evidências identificadas nas } \\
\text { conclusões dos estudos }\end{array}$ \\
\hline $\begin{array}{l}\text { BIASIBETTI et al. } \\
(2019)\end{array}$ & $\begin{array}{l}\text { Comunicação para a } \\
\text { segurança do paciente } \\
\text { em internações } \\
\text { pediátricas }\end{array}$ & $\begin{array}{l}\text { Analisar a percepção de profissionais } \\
\text { de saúde e acompanhantes/familiares } \\
\text { quanto ao desenvolvimento da } \\
\text { comunicação para a segurança do } \\
\text { paciente em internações pediátricas. } \\
\text { Estudo exploratório-descritivo, } \\
\text { qualitativo. }\end{array}$ & $\begin{array}{l}\text { "Barreiras para a Comunicação Efetiva" } \\
\text { que abordou as falhas e dificuldades no } \\
\text { processo de comunicação e } \\
\text { "Ferramentas para Qualificar a } \\
\text { Comunicação" que apresenta } \\
\text { recomendações para as melhorias, em } \\
\text { especial, instrumentalização do } \\
\text { acompanhante/familiar. }\end{array}$ \\
\hline PERES et al. (2019) & $\begin{array}{l}\text { Percepção de } \\
\text { familiares e cuidadores } \\
\text { quanto à } \\
\text { segurança do paciente } \\
\text { em unidades de } \\
\text { internação pediátrica }\end{array}$ & $\begin{array}{l}\text { Conhecer a percepção de familiares e } \\
\text { cuidadores quanto à Segurança do } \\
\text { Paciente em unidades de internação } \\
\text { pediátrica. Estudo qualitativo } \\
\text { exploratório-descritivo. }\end{array}$ & $\begin{array}{l}\text { As percepções dos cuidadores } \\
\text { referentes à segurança do paciente em } \\
\text { unidades de internação pediátrica } \\
\text { demonstram que estes absorvem } \\
\text { orientações que favorecem o cuidado } \\
\text { seguro, embora não tenham um } \\
\text { conhecimento formal a respeito do } \\
\text { assunto. }\end{array}$ \\
\hline $\begin{array}{l}\text { RODRIGUES et al. } \\
(2018)\end{array}$ & $\begin{array}{l}\text { Segurança do Paciente } \\
\text { em Unidade Neonatal: } \\
\text { Preocupações e } \\
\text { estratégias vivenciadas } \\
\text { por Pais. }\end{array}$ & $\begin{array}{l}\text { Analisar como os pais identificam a } \\
\text { segurança do paciente em unidade } \\
\text { neonatal. Estudo de caso exploratório, } \\
\text { com abordagem qualitativa. }\end{array}$ & $\begin{array}{l}\text { Apesar de inquietações, os pais sentem- } \\
\text { se seguros em relação à assistência, } \\
\text { considerando que houve mais } \\
\text { estratégias identificadas do que } \\
\text { preocupações. }\end{array}$ \\
\hline PEREIRA & O Papel do Paciente & Apresentar os dados analisados na & Aspectos como gestão do tratamento e \\
\hline
\end{tabular}




\begin{tabular}{|c|c|c|c|}
\hline$(2018)$ & \begin{tabular}{lr}
\multicolumn{2}{l}{ como Coprodutor nos } \\
serviços de & Saúde: \\
Uma & revisão \\
sistemática & de \\
literatura. &
\end{tabular} & $\begin{array}{l}\text { literatura científica sobre a atuação do } \\
\text { paciente como coprodutor nos serviços } \\
\text { de saúde. Revisão sistemática de } \\
\text { literatura. }\end{array}$ & $\begin{array}{l}\text { os impactos da coprodução na cadeia } \\
\text { de valor dos serviços de saúde ainda } \\
\text { não são tratados de forma aprofundada } \\
\text { pela literatura. }\end{array}$ \\
\hline $\begin{array}{l}\text { BANDEIRA et al. } \\
(2017)\end{array}$ & \begin{tabular}{lr}
\multicolumn{2}{l}{ Condutas de educação } \\
ao familiar $r$ para \\
promoção & da \\
segurança da & criança \\
hospitalizada: & registros \\
da & equipe \\
multiprofissional.
\end{tabular} & $\begin{array}{l}\text { Analisar os registros da equipe } \\
\text { multiprofissional sobre condutas de } \\
\text { educação do familiar para promoção da } \\
\text { segurança da criança hospitalizada. } \\
\text { Estudo transversal, descritivo e } \\
\text { retrospectivo com uso de dados } \\
\text { secundários. }\end{array}$ & $\begin{array}{l}\text { As informações relacionadas às } \\
\text { condutas de educação do familiar para } \\
\text { promover a segurança do paciente } \\
\text { pediátrico ainda são pouco registradas } \\
\text { em prontuário, sendo necessário } \\
\text { envolver a equipe multiprofissional } \\
\text { nesse processo. }\end{array}$ \\
\hline GOMES et al. (2017) & $\begin{array}{lr}\text { Identificação } & \text { do } \\
\text { paciente } & \text { em } \\
\text { neonatologia } & \text { para } \\
\text { assistência segura. }\end{array}$ & $\begin{array}{l}\text { Analisar a identificação de pacientes } \\
\text { neonatos por meio de pulseira. Estudo } \\
\text { transversal, observacional, documental, } \\
\text { com abordagem quantitativa. }\end{array}$ & $\begin{array}{l}\text { A unidade investigada apresenta falhas } \\
\text { relacionadas ao processo de } \\
\text { identificação de seus pacientes, } \\
\text { indicando que a implementação de } \\
\text { estratégias para assistência segura é } \\
\text { necessária e urgente. }\end{array}$ \\
\hline $\begin{array}{l}\text { BITTENCOURT et al. } \\
(2017)\end{array}$ & $\begin{array}{l}\text { Fatores associados ao } \\
\text { risco de quedas em } \\
\text { pacientes adultos } \\
\text { hospitalizados*. }^{*}\end{array}$ & $\begin{array}{l}\text { Analisar os fatores relacionados ao } \\
\text { risco de quedas em pacientes adultos } \\
\text { internados em um hospital. Estudo } \\
\text { quantitativo, transversal e analítico, } \\
\text { desenvolvido em unidades de } \\
\text { Internação Clínica e Cirúrgica de um } \\
\text { Hospital Privado, de meio porte, na } \\
\text { região noroeste do estado do Rio } \\
\text { Grande do Sul. }\end{array}$ & $\begin{array}{l}\text { Associação entre o risco de quedas com } \\
\text { motivo de internação, comorbidades e } \\
\text { fatores intrínsecos. Em relação aos } \\
\text { fatores extrínsecos, foi encontrada } \\
\text { associação entre tapetes e risco de } \\
\text { quedas. Não houve associação entre o } \\
\text { risco de quedas com outros fatores } \\
\text { extrínsecos. }\end{array}$ \\
\hline $\begin{array}{l}\text { ARRUDA et al. } \\
(2017)\end{array}$ & $\begin{array}{l}\text { Percepção do paciente } \\
\text { com a segurança no } \\
\text { atendimento em } \\
\text { unidade de urgência e } \\
\text { emergência. }\end{array}$ & $\begin{array}{l}\text { Analisar a percepção do paciente } \\
\text { quanto à segurança no atendimento em } \\
\text { saúde, oferecida pelos profissionais em } \\
\text { unidade de urgência e emergência } \\
\text { hospitalar. Estudo quanti-qualitativo, } \\
\text { transversal, descritivo, realizado com } \\
100 \text { pacientes atendidos na unidade de } \\
\text { urgência e emergência de um hospital } \\
\text { de ensino do Centro-Oeste brasileiro } \\
\text { por meio de instrumento } \\
\text { semiestruturado. }\end{array}$ & $\begin{array}{l}\text { Alto nível de satisfação dos pacientes } \\
\text { em relação à segurança no atendimento } \\
\text { e necessidade de a instituição centrar } \\
\text { seus objetivos em um sistema de } \\
\text { avaliação da qualidade assistencial. }\end{array}$ \\
\hline AN et al. (2017) & $\begin{array}{l}\text { The effects of patient } \\
\text { education on patient } \\
\text { safety: can we change } \\
\text { patient perceptions and } \\
\text { attitudes?: Lessons } \\
\text { from the Armed Forces } \\
\text { Capital Hospital in } \\
\text { Korea. }\end{array}$ & $\begin{array}{l}\text { Avaliar os efeitos da educação em } \\
\text { segurança nas percepções e atitudes } \\
\text { do paciente em relação à segurança no } \\
\text { pessoal militar. Estudo quase } \\
\text { experimental. }\end{array}$ & $\begin{array}{l}\text { A educação do paciente foi associada a } \\
\text { escores mais altos tanto na percepção } \\
\text { quanto nas atitudes sobre segurança. } \\
\text { Para melhorar o envolvimento do } \\
\text { paciente nessa área, métodos que } \\
\text { incentivem o empoderamento do } \\
\text { paciente devem ser desenvolvidos. }\end{array}$ \\
\hline YAN et al. (2017) & $\begin{array}{l}\text { Patient reporting of } \\
\text { undesirable events: a }\end{array}$ & $\begin{array}{l}\text { Identificar a frequência de eventos } \\
\text { indesejáveis relatados pelos pacientes }\end{array}$ & $\begin{array}{lccr}\text { Realização } & \text { de } & \text { levantamento } & \text { de } \\
\text { pacientes } & \text { para } & \text { investigar } & \text { suas }\end{array}$ \\
\hline
\end{tabular}




\begin{tabular}{|c|c|c|c|}
\hline & pilot study in China. & $\begin{array}{l}\text { durante a hospitalização e explorar a } \\
\text { relação entre eventos indesejáveis e } \\
\text { percepção de segurança e satisfação } \\
\text { com o cuidado na China. Levantamento } \\
\text { transversal. }\end{array}$ & $\begin{array}{l}\text { experiências pessoais de eventos } \\
\text { relacionados à segurança é uma } \\
\text { abordagem promissora e uma } \\
\text { ferramenta valiosa para identificar e } \\
\text { monitorar áreas problemáticas de } \\
\text { atendimento. }\end{array}$ \\
\hline KEVIN et al. (2017) & $\begin{array}{l}\text { Preventing Harm in the } \\
\text { ICU - Building a } \\
\text { Culture of Safety - } \\
\text { Patients and Families. }\end{array}$ & $\begin{array}{l}\text { Descrever o papel crítico de segurança } \\
\text { e do envolvimento do paciente e da } \\
\text { família em iniciativas bem-sucedidas de } \\
\text { melhoria da qualidade na UTI. Revisão } \\
\text { de literatura. Pesquisas dos recursos } \\
\text { impressos e baseados na Web das } \\
\text { principais organizações de segurança } \\
\text { do paciente. }\end{array}$ & $\begin{array}{l}\text { Os esforços para estabelecer uma } \\
\text { cultura de segurança e envolver } \\
\text { significativamente os pacientes e } \\
\text { familiares devem formar a base para } \\
\text { todas as intervenções de segurança na } \\
\text { UTI. }\end{array}$ \\
\hline $\begin{array}{l}\text { FASSARELLA et al. } \\
(2017)\end{array}$ & $\begin{array}{l}\text { Profissionais } \\
\text { mediadores da } \\
\text { qualidade e segurança } \\
\text { do paciente como } \\
\text { estratégia para o } \\
\text { cuidado seguro. }\end{array}$ & $\begin{array}{l}\text { Identificar as ações e atribuições dos } \\
\text { mediadores frente às estratégias } \\
\text { implantadas pelo gestor de risco para } \\
\text { melhoria dos cuidados de saúde. } \\
\text { Estudo transversal, descritivo e } \\
\text { exploratório, de abordagem } \\
\text { quantitativa. }\end{array}$ & $\begin{array}{l}\text { Notou-se a pertinência da iniciativa } \\
\text { como uma medida inovadora e pioneira } \\
\text { e no aspecto da tecnologia leve em } \\
\text { saúde, embora não referida pelos } \\
\text { participantes. Percebeu-se evolução } \\
\text { nas relações entre os membros da } \\
\text { equipe e da produção de comunicação. }\end{array}$ \\
\hline PEDRO et al. (2016) & $\begin{array}{l}\text { Conhecimento do } \\
\text { paciente sobre a } \\
\text { assistência hospitalar } \\
\text { recebida durante sua } \\
\text { internação. }\end{array}$ & $\begin{array}{l}\text { Identificar o conhecimento do paciente } \\
\text { sobre a sua situação de saúde e } \\
\text { assistência hospitalar correlata durante } \\
\text { o período de internação. Estudo } \\
\text { descritivo, transversal, quantitativo. }\end{array}$ & $\begin{array}{l}\text { O conhecimento do paciente em regime } \\
\text { hospitalar é, ainda, um fenômeno } \\
\text { deficitário, uma vez que se limita apenas } \\
\text { aos aspectos mais básicos de seu } \\
\text { problema de saúde, corroborando a sua } \\
\text { passividade na atenção e, } \\
\text { possivelmente, reduzindo sua } \\
\text { participação no cuidado. }\end{array}$ \\
\hline $\begin{array}{l}\text { BEZERRA et al. } \\
(2016)\end{array}$ & \begin{tabular}{l} 
Conhecimentos \\
\multicolumn{2}{l}{ de } \\
usuários de uma clínica \\
cirúrgica sobre $\quad$ a \\
ocorrência \\
incidentes.
\end{tabular} & $\begin{array}{l}\text { Identificar a ocorrência de incidentes } \\
\text { percebidos pelos pacientes durante o } \\
\text { período de internação hospitalar, } \\
\text { analisar a opinião dos usuários sobre a } \\
\text { ocorrência de incidentes e classificar os } \\
\text { incidentes percebidos quanto ao tipo, } \\
\text { causas e consequências. Estudo } \\
\text { descritivo, transversal. }\end{array}$ & $\begin{array}{l}\text { Apesar de inseridos no contexto da } \\
\text { assistência, muitos profissionais ainda } \\
\text { não reconhecem a importância do } \\
\text { envolvimento dos usuários para a } \\
\text { prevenção de incidentes, necessitando } \\
\text { de ações educativas, com foco na } \\
\text { segurança do paciente, para o } \\
\text { empoderamento dos usuários (AU). }\end{array}$ \\
\hline SILVA et al. (2016) & $\begin{array}{l}\text { O envolvimento do } \\
\text { paciente na segurança } \\
\text { do cuidado: revisão } \\
\text { integrativa. }\end{array}$ & $\begin{array}{l}\text { Levantar as estratégias adotadas pelas } \\
\text { instituições de saúde, que envolvem o } \\
\text { paciente no cuidado, como barreira } \\
\text { para a prevenção de incidentes. } \\
\text { Revisão integrativa de literatura. }\end{array}$ & $\begin{array}{l}\text { A avaliação ou descrição de práticas } \\
\text { institucionais que envolvem o paciente } \\
\text { na sua segurança emerge como lacuna } \\
\text { do conhecimento científico. O impacto } \\
\text { dessa revisão está em constatar a } \\
\text { necessidade de estudos randomizados } \\
\text { para identificar intervenções efetivas, } \\
\text { direcionando as instituições de saúde } \\
\text { para a mudança da cultura } \\
\text { organizacional, com foco na segurança } \\
\text { e no cuidado centrado no paciente. }\end{array}$ \\
\hline
\end{tabular}




\begin{tabular}{|c|c|c|c|}
\hline & $\begin{array}{l}\text { Análise de vídeos do } \\
\text { YouTube sobre eventos } \\
\text { adversos em saúde. }\end{array}$ & $\begin{array}{l}\text { Analisar os vídeos do YouTube sobre } \\
\text { eventos adversos em saúde, } \\
\text { identificando a existência de relações } \\
\text { com a segurança do paciente. } \\
\text { Pesquisa do tipo exploratória, com } \\
\text { abordagem quantitativa. }\end{array}$ & $\begin{array}{l}\text { Existência de um número incipiente de } \\
\text { vídeos frente à relevância da temática } \\
\text { na atualidade, mas que destacam a } \\
\text { relação entre a segurança do paciente e } \\
\text { os eventos adversos em saúde, com } \\
\text { enfoque nas estratégias de prevenção. }\end{array}$ \\
\hline SOUZA & $\begin{array}{l}\text { Estudo exploratório das } \\
\text { iniciativas acerca da } \\
\text { segurança do paciente } \\
\text { em hospitais do Rio de } \\
\text { Janeiro. }\end{array}$ & $\begin{array}{l}\text { Analisar, com base nas informações } \\
\text { obtidas com os gerentes de risco, as } \\
\text { iniciativas implementadas para garantir } \\
\text { a segurança do paciente. Estudo } \\
\text { exploratório com abordagem } \\
\text { quantitativa dos dados. }\end{array}$ & $\begin{array}{l}\text { Todos os profissionais implementam a } \\
\text { identificação dos pacientes, mas os } \\
\text { gerentes de risco desenvolvem } \\
\text { iniciativas que demandam menos } \\
\text { investimentos e têm suas ações } \\
\text { direcionadas principalmente para a } \\
\text { educação continuada, contrário ao que é } \\
\text { proposto atualmente. }\end{array}$ \\
\hline $\begin{array}{l}\text { WEGNER E PEDRO } \\
(2012)\end{array}$ & $\begin{array}{l}\text { A segurança do } \\
\text { paciente nas } \\
\text { circunstâncias de } \\
\text { cuidado: prevenção de } \\
\text { eventos adversos na } \\
\text { hospitalização infantil. }\end{array}$ & $\begin{array}{l}\text { Analisar como as } \\
\text { acompanhantes/cuidadoras } \\
\text { profissionais da saúde reconhecem os } \\
\text { eventos adversos nas circunstâncias de } \\
\text { cuidado. Pesquisa de abordagem } \\
\text { qualitativa do tipo estudo de caso. }\end{array}$ & $\begin{array}{l}\text { Recomenda-se mudanças na cultura } \\
\text { organizacional das instituições de saúde } \\
\text { para uma cultura de segurança, com } \\
\text { ênfase no cuidado seguro em saúde e } \\
\text { visão sistêmica na avaliação da } \\
\text { ocorrência de eventos adversos. }\end{array}$ \\
\hline
\end{tabular}

Fonte: Dados da pesquisa, 2019.

O Quadro 1 evidencia e resume as principais estratégias analisadas como vantajosas pelos autores para envolver o paciente e sua família no processo de cuidar, a saber: Quatro Es (THORNTON et al., 2017); Grupo de mediadores (FASSARELLA et al., 2017); Trabalho interdisciplinar (WEGNER; PEDRO, 2012); Envolvimento dos pacientes no autocuidado (YAN et al., 2017; THORNTON et al., 2017; BEZERRA et al., 2016; PEDRO et al., 2016);Programa educacional para os pacientes (AN et al., 2017); Atenção centrada no paciente (BRASIL, 2017b); Melhoria da comunicação com o paciente/familiar (BEZERRA et al., 2016; WEGNER; PEDRO, 2012; RODRIGUES et al., 2018 SILVA et al., 2016, BIASIBETTI et al., 2019); e Utilização de vídeos e cartilhas como material educativo (SALVADOR et al., 2014; SILVA et al., 2016; COSTA; BORCHARDT; PEREIRA, 2018; BIASIBETTI et al., 2019).

Os artigos analisados denotam a importância da adoção de tais práticas nas unidades de saúde e enfocam a participação do paciente, cuidador e profissional da área em questão. Além disso, apontam a necessidade de aplicar e ampliar os processos e protocolos propostos por órgão nacionais, como a Agência de Vigilância Sanitária - Anvisa, e internacionais, como a OMS.

\section{DISCUSSÃO}




\section{Estratégia da Cultura de Segurança}

A cultura de segurança perpassa pela capacitação de profissionais da área da saúde de linha de frente, da equipe, dos pacientes e de familiares, com o intuito de melhorar o processo do cuidado (THORNTON et al. 2017).

Thornton et al. (2017) utilizaram como estratégia para estabelecer uma cultura de segurança o modelo do Projeto Michigan Keystone em uma UTI. Tal projeto utilizava os quatro Es (Envolva, Eduque, Execute e Avalie), transportados para a Cultura de Segurança.

O primeiro E, Envolva, consiste na ideia de envolver os interessados, isto é, fazer com que ocorra o engajamento de médicos, funcionários de linha de frente, para gerentes de nível médio para o executivo, com intuito de criar uma cultura de segurança duradoura. A partir do momento em que a cultura de segurança se encontra consolidada, denota-se que os profissionais estão engajados na melhoria da segurança do paciente (FASSARELLA et al., 2017).

No tocante ao Eduque, uma opção é utilizar materiais para treinamento, oferecidos tanto online quanto impressos, que abarquem a comunicação, as habilidades de trabalho em equipe, bem como o achatamento das hierarquias rígidas. Assim, é possível propiciar vários pontos de vista e promover o respeito a todos os envolvidos: colegas, subordinados, pacientes e familiares (THORNTON et al., 2017).

A variável Execução é de extrema valia, pois evita danos e busca a melhoria dos processos da cultura de segurança do paciente.

Quanto à Avaliação, os impactos específicos são importantes para que se possa alcançar e manter uma cultura de segurança, propiciando melhoria nos resultados. O sistema para melhoria da cultura de segurança deve incluir "[...] governança, liderança, gerenciamento, tecnologia de informação, capacitação, desenvolvimento de recursos e aprendizagem, todos trabalhando juntos para garantir a segurança" (THORNTON et al., 2017, p. 5).

Fassarella et al. (2017, p. 6) corroboram os autores supracitados e afirmam que:

Para se criar uma cultura de segurança, significa vencer barreiras e desenvolver um ambiente de trabalho colaborativo, no qual os membros da equipe assistencial tratam um ao outro como iguais e com respeito, independentemente da função de trabalho ou título. Envolve impor responsabilidade pessoal e organizacional.

A Cultura da Segurança envolve mudança na cultura da organização, de forma que haja o engajamento dos pacientes e familiares, oferecendo-se, assim, impacto substancial em todo o processo. O incentivo e a participação dos familiares no cuidado promovem a cultura de 
segurança e permite a aproximação dos profissionais de saúde, favorecendo a adesão ao tratamento e autonomia do paciente (PERES et al. 2019).

Fassarella et al. (2017) relatam e analisam práticas para o fortalecimento da cultura de segurança. A estratégia apresentada pelos autores foi a implantação de um grupo de mediadores em um hospital de Portugal. Os mediadores são colaboradores escolhidos que farão o elo entre as unidades assistenciais e o escritório da qualidade, tais como:

[...] disseminação da cultura de segurança, efetividade da comunicação interprofissional, distribuição do conhecimento e da informação direcionada para a segurança e de intervenções voltadas para problemas específicos, melhorando à assistência à saúde em todos os pontos de cuidados de forma integrada, participativa, coletiva e em um processo de melhoria contínua (FASSARELLA et al., 2017, p. 2).

Com relação às orientações de segurança do paciente nos prontuários médicos, Bandeira et al. (2017) relatam que 100\% dos registros foram feitos pela equipe de enfermagem e ressaltam a necessidade de incluir os demais profissionais na corresponsabilização pelos processos de segurança do paciente. Para tanto, é necessário o desenvolvimento de atitudes proativas em prol da segurança, e tais ações devem ocorrer na prática e em curto prazo de tempo.

Nesse sentido, a REBRAENSP (2013, p. 96) elenca que "a parceria entre a equipe de saúde e o paciente/acompanhante implica a aprendizagem de novas perspectivas e comportamentos para todos os envolvidos, exigindo paciência e sensibilidade da equipe multiprofissional". No entanto, é imprescindível o desenvolvimento de estratégias de apoio educacional para sustentar as práticas seguras e baseadas em evidência, de maneira que haja o envolvimento dos profissionais e o comprometimento dos gestores em todos os processos.

Um dos fatores determinantes para o desenvolvimento da cultura de segurança é a adoção de processos e diretrizes para estimular o trabalho interdisciplinar e de colaboração pelas instituições de saúde. O trabalho interdisciplinar, assim, é uma sugestão para avançar na segurança do paciente e na qualidade da atenção à saúde (WEGNER; PEDRO, 2012).

A China é exemplo de um país que buscou esforços para melhorar a segurança do paciente, uma vez que o monitoramento dos pacientes era bastante rudimentar. Com isso, ela se modernizou e se atualizou frente à segurança do paciente, participando da oficina da Organização Mundial de Saúde (OMS), "Pacientes para a Segurança do Paciente", devido ao alto número de eventos relacionados à segurança. A estratégia adotada buscou envolver os pacientes em atividades orientadas para que pudessem desenvolver o autocuidado, pois são eles que estão no centro do processo de cuidado de saúde. Tais ações permitiram motivar os pacientes de maneira eficaz para um tratamento seguro (YAN et al., 2017). 
An et al. (2017), por sua vez, realizaram um estudo no Hospital das Forças Armadas da Coreia e compararam dois grupos: um grupo que recebeu orientações sobre a segurança do paciente e outro grupo que não recebeu. Identificou-se que os pacientes que participaram do programa educacional aumentaram a percepção e atitudes que refletem na segurança do paciente. Na Análise de Subgrupos, os autores observaram que a mudança de atitudes está relacionada ao estado de saúde do indivíduo. "Alguns pacientes podem requerer mais intervenção para serem motivados a se envolver em segurança" (AN et al., 2017, p. 395).

O envolvimento do paciente no processo de cultura de segurança é de extrema importância, pois possibilita que haja o engajamento no autocuidado, de maneira que os pacientes tomem conhecimento sobre o seu estado de saúde, bem como possam inferir nas decisões, o que minimiza a ocorrência de incidentes. Tais assertivas são propiciadas por meio da educação e da participação, o que tornará o paciente corresponsável pelo seu tratamento (THORNTON et al., 2017; BEZERRA et al., 2016; PEDRO et al., 2016).

Uma das dimensões da qualidade dos serviços de saúde, segundo a Anvisa, está na atenção centrada no paciente, que "envolve o respeitar o paciente, considerando suas preferências individuais, necessidades e valores, assegurando que a tomada de decisão clínica se guiará por tais valores" (BRASIL, 2017b, p. 32).

Um dos desafios elencados no documento de referência ao PNSP é a estratégia da disseminação da cultura de segurança, capaz de propiciar uma "[...] ação de comunicação social ampla para que a busca pela segurança do paciente passe a ser de domínio público" (BRASIL, 2014, p. 14). Além disso, o documento reforça a importância de se criar um ambiente favorável a mudanças, com manutenção efetiva da segurança do paciente. Portanto, é imprescindível colocar o paciente como fator-chave nos processos de segurança e melhora dos cuidados de saúde.

\section{Estratégia de comunicação}

A comunicação é o basilar entre os profissionais de saúde, pacientes e familiares (BEZERRA et al., 2016; WEGNER; PEDRO, 2012; RODRIGUES et al., 2016; SILVA et al., 2016). Segundo Biasibetti (2019), os acompanhantes e familiares valorizam a comunicação como ponto positivo durante a hospitalização. No entanto, várias são as lacunas que existem nesse processo. Alguns dos artigos selecionados descrevem a percepção dos usuários sobre a temática segurança e demonstram a fragilidade que se destaca na comunicação entre as partes. 
Wegner e Pedro (2012) detectaram que a comunicação efetiva é imprescindível para a qualidade de saúde da criança hospitalizada. Essa questão pode ser exemplificada na fala de uma das acompanhantes entrevistadas no estudo dos autores: "a atenção dos enfermeiros, a atenção dos médicos, de toda a equipe, não tem o que se queixar e qualquer coisa que a gente precise eles tão atentos pra nos responder, satisfazer nossas perguntas, o que a gente precisa (A5)" (WEGNER; PEDRO, 2012, p. 4)

Também no estudo de Rodrigues et al. (2018) foram realizadas entrevistas nas quais os participantes relataram: "tudo é muito bem conversado [...] e mesmo assim passado para mim. (M1)" e "ele [médico] nos passa aquela segurança, aquela coisa que todo o pai e toda a mãe quer ouvir. (M7)" (RODRIGUES; et al., 2018, p. 4).

Nessa perspectiva, para que a comunicação efetiva ocorra, é necessário que haja clareza e eficácia. As informações devem estar completas e precisas, de maneira aberta, propiciando uma relação mais efetiva entre profissional e paciente.

[...] a comunicação é uma ferramenta de extrema importância para a efetiva relação entre profissional e paciente e que contribui, diretamente, para a prevenção de incidentes, além de ser apontada como uma questão ética no contexto da assistência à saúde (SILVA et al., 2016, p. 8).

De forma geral, a partir dos estudos que relatam e analisam a prática, nota-se ainda a fragilidade nas condutas para ampliar a segurança do paciente. Apesar de se reconhecer e valorizar a importância do empoderamento e do envolvimento paciente/familiares, esses ainda são fatores limitantes nas instituições. É de extrema importância melhorar o mecanismo de comunicação e buscar estratégias para alcançar esse objetivo.

\section{Outras estratégias: vídeos/cartilha}

Para estimular a participação do paciente e seus familiares no processo do cuidado é muito importante a disponibilização de documentos apropriados que sejam capazes de instrumentalizá-los neste sentido (BRASIL, 2017b). Por isso, a utilização de técnicas e instrumentos como estratégia para uma comunicação efetiva sobre o paciente e seus cuidados ampliam a concepção para que o paciente e seu cuidador tenham voz para colaborar neste processo (BIASIBETTO, 2019).

Em consequência, é importante referir-se a outra estratégia bastante relevante que pode auxiliar na disseminação da cultura de segurança, que é a utilização de materiais como vídeos e cartilhas. 
Salvador et al. (2014) dedicaram-se a avaliar a utilização de vídeos. Para tanto, os autores investigaram os vídeos disponibilizados no sítio de compartilhamento do YouTube, buscando identificar a existência de relações com a segurança do paciente frente aos eventos adversos.

\begin{abstract}
Como elemento fundamental da promoção da cultura de segurança do paciente, os vídeos destacaram o paciente como um elemento fundamental no processo de prevenção de eventos adversos, o qual deve participar efetivamente dos cuidados de segurança que the serão benéficos. Destaca-se que o envolvimento do paciente para garantir sua própria segurança é recomendado, constituindo-se a última barreira para a interceptação de um incidente, bem como um importante avaliador da segurança e qualidade da assistência recebida (SALVADOR et al. 2014, p. 836).
\end{abstract}

Ainda no que diz respeito à utilização de vídeos como meio de disseminação da cultura de segurança, Silva et al. (2016) ressaltam que a orientação aos pacientes, pais e/ou cuidadores incentiva a sua participação tanto na fiscalização quanto na vigilância dos cuidados. Portanto, esses autores perceberam que a linguagem deve ser precisa e única, evitando-se distorções nas informações sobre a predisposição a um evento adverso em saúde.

Outra estratégia é o uso de cartilhas que possibilitam o entendimento do paciente acerca de sua patologia, além de orientar em prol de dirimir dúvidas a respeito do seu problema de saúde.

A importância de os pacientes serem tratados como parceiros, com corresponsabilidade e vínculos solidários, nos esforços para prevenir todo mal evitável em saúde, percorre os programas: Paciente pela Segurança do Paciente, da OMS, Política Nacional de Humanização e Documento de Referência da PNSP.

\begin{abstract}
Talvez, esse seja o eixo mais difícil a ser desenvolvido, pois envolve uma grande mudança de cultura nos estabelecimentos de Saúde. O grande desafio será combinar ações desenvolvidas pelos NSPs, conselhos profissionais, órgãos de classe e gestores com aquelas que ampliem o acesso da sociedade às informações relativas à segurança do paciente (BRASIL, 2014, p. 27)
\end{abstract}

A importância da participação ativa dos pacientes e familiares durante o seu tratamento vem sendo reconhecida nos últimos anos pelos serviços de saúde. É fundamental que esses sujeitos estejam devidamente educados para atuarem como coprodutores (COSTA; BORCHARDT; PEREIRA, 2018). Em consonância com os autores, então, acredita-se que o uso de cartilhas e vídeos pode ser viável para o processo de instrução a respeito dessas questões. Principalmente, quando utilizados como suporte ao processo de educação ao cuidado. 


\title{
Estratégias para a segurança do paciente
}

As Metas Internacionais de Segurança do Paciente são: identificar corretamente os pacientes; melhorar a comunicação entre profissionais de saúde; melhorar a segurança na prescrição, no uso e na administração de medicamentos; assegurar cirurgias com local de intervenção, procedimento e paciente correto; higienizar as mãos para evitar infecções e reduzir o risco de queda e úlceras por pressão. (BRASIL, 2013).

Os documentos analisados ressaltam a importância do estudo e da implantação de estratégias que visem à segurança do paciente. Dentre elas, destacam-se: a identificação do paciente, a higienização das mãos e a prevenção de quedas.

Para avaliar tais metas, Arruda et al. (2017) efetuaram um estudo na unidade de emergência e urgência do Hospital de Ensino do Centro-Oeste. Os autores detectaram que os profissionais possuem condutas seguras, tais como: confirmar o nome do paciente, explicar e orientar sobre os procedimentos e as complicações que podem surgir devido ao procedimento, consultar a prescrição, entre outros. Neste sentido, ao ser informado sobre o seu trabalho, o paciente sente segurança nos profissionais envolvidos, gerando, assim, satisfação no cuidado dispensado.

\section{Identificação do paciente}

A identificação do paciente é de extrema importância, pois assegura a qualidade e segurança do cuidado no serviço de saúde, conforme é preconizado pelas metas internacionais de segurança do paciente (SOUZA; SILVA, 2014).

\begin{abstract}
Dentro das metas internacionais para a segurança do paciente, a identificação do paciente é a primeira meta proposta e tem como principais medidas: enfatizar a responsabilidade dos profissionais de saúde em verificar a identificação dos pacientes antes da realização de cuidados, identificar os pacientes com pelo menos dois identificadores, por exemplo, nome do paciente e data de nascimento; implementar protocolos para a identificar pacientes com mesmo nome, em coma ou confusos; encorajar os pacientes a participar de todas as etapas do processo, entre outros (SOUZA; SILVA, 2014, p. 25).
\end{abstract}

Gomes et al. (2017) relatam que, em estudo efetuado no Hospital Escola de Santa Catarina, detectou-se que em 81 dos 223 procedimentos de administração de medicamentos e hemoderivados (36,2\%), "não houve nenhum tipo de identificação do paciente" (GOMES et al., 2017 p. 7). Ressalta-se que a ausência das pulseiras pode gerar erros de medicação ou hemoderivados, troca de bebês, entre outros. 
Em outra pesquisa realizada por Rodrigues et al. (2018), em uma Unidade de Internação Neonatal de um hospital público da região Sul do Brasil, um familiar participante da pesquisa declarou: "quem não chama pelo nome não cuida" (RODRIGUES et al., 2018). Tal assertiva denota a responsabilidade de todos os envolvidos na área de saúde, como também a necessidade de fortalecimento do processo de identificação, evitando-se, assim, eventos adversos.

No estudo realizado por Peres et al. (2019) percebemos melhora no requisito no qual os acompanhantes relataram que a equipe assistencial confere $o$ nome do paciente e a integridade da pulseira de identificação, mas deixavam de realizar esta conferência quando a internação se torna prolongada. Isso indica ser necessário consolidar os procedimentos de conferência de identificação do paciente, mesmo quando se tratar de pacientes já conhecidos da equipe assistencial.

\section{Higienização das mãos}

A higienização das mãos é uma prática extremamente necessária e importante no cuidado com a saúde. Por meio dessa ação é possível evitar diversas infecções adquiridas no ambiente hospitalar.

Dentre os entrevistados por Rodrigues et al. (2018), um dos familiares expôs que: "tem que lavar as mãos, tem que higienizar". Tal atitude vai ao encontro das diretrizes para segurança do paciente. De acordo com Brasil (2017a), são cinco os momentos para a higienização das mãos: antes do contato com o paciente, antes da realização de procedimento asséptico, após risco de exposição a fluidos corporais, após contato com o paciente e após contato com as áreas próximas ao paciente.

No estudo realizado por Peres et al. (2019) os acompanhantes e familiares relatam receber orientações quanto à forma correta e às razões para a higienização das mãos. Entretanto, observaram que nem sempre os profissionais higienizam as mãos nos momentos recomendados.

A adoção e orientação dessa medida preventiva são de responsabilidade da equipe multidisciplinar de saúde, o que proporciona a redução do número de infecções associadas ao cuidado em saúde. A partir da orientação feita à família e ao paciente, estes começam a entender a importância e a demandar para a equipe assistencial a correta higienização das mãos. 


\section{Quedas}

Um dos eventos adversos que as instituições de saúde buscam dirimir é a queda dos pacientes, sendo este um dos indicadores da qualidade dos serviços prestados. Estudos internacionais, segundo aponta Brasil (2017a), detectaram que quanto maior o quadro de pessoal da enfermagem, ou horas de enfermagem por paciente, menor é o índice de quedas.

As quedas, por serem eventos capazes de ocasionar complicações e consequente aumento no tempo de internação, elevação de custos hospitalares, morbidade e mortalidade, afetam também a confiança do paciente e de seus familiares (BITTENCOURT et al., 2017; BRASIL, 2017a).

A falta de pisos antiderrapantes, de barras no banheiro e no quarto do paciente e de grades no leito são variáveis que podem contribuir para a ocorrência de quedas. Para evitá-las, BRASIL (2017a, p. 71) recomenda:

[...] a utilização de pisos antiderrapantes, instalação de lâmpadas de segurança nos banheiros e corredores, uso de dispositivos de auxílio para deambulação com supervisão, instalação de barras de apoio no banheiro e no chuveiro, manutenção de grades no leito do paciente e reforço de orientações para os acompanhantes dos pacientes com diagnóstico de risco para quedas.

É sabido que a hospitalização aumenta o risco de queda (LUIZA et al., 2019). Para evitar tal transtorno, é necessário adotar medidas em que estarão envolvidos tanto os familiares quanto a equipe multidisciplinar, tais como programas de educação continuada para a equipe, para acompanhantes e para pacientes, potencializando, assim, a segurança do paciente.

\section{CONSIDERAÇÕES FINAIS}

A Cultura de Segurança busca propiciar aos profissionais envolvidos mais respeito, responsabilidade organizacional e pessoal. Também, oferece aos pacientes e familiares um cuidado mais seguro, pois consolida a comunicação mais efetiva, assim como resulta na melhoria da assistência à saúde e do comprometimento dos gestores envolvidos.

Os autores analisados indicaram diversificadas estratégias delineadas, dentre elas: quatro Es, grupo de mediadores, trabalho interdisciplinar, envolvimento dos pacientes no autocuidado, programa educacional para os pacientes, atenção centrada no paciente, melhoria da comunicação com o paciente/familiar e utilização de vídeos e cartilhas como material educativo. Todas essas estratégias mostraram-se efetivas para propiciar segurança ao paciente e qualidade da atenção à saúde. 
A técnica de envolver e engajar o paciente no autocuidado desenvolve maior motivação para o devido tratamento e, assim, aumenta a sua percepção e participação durante o processo. Para tanto, a comunicação entre os profissionais, pacientes, familiares e acompanhantes deve ser clara, precisa, completa e efetiva, pois, além de esclarecer as dúvidas, pode também ajudar a evitar incidentes.

Os vídeos e as cartilhas são grandes aliados na disseminação da cultura de segurança, uma vez que, além de ensinar, incitam os pacientes, familiares e/ou cuidadores a participarem dos cuidados necessários. Ao fornecerem as informações relativas à segurança do paciente, propiciam também que esses usuários participem ativamente do tratamento, sendo coprodutores do processo.

Foram avaliadas as metas internacionais de identificação do paciente, higienização das mãos e quedas. Verificou-se a importância de cada uma delas, porém, para que essas metas sejam validadas, o paciente e o familiar/acompanhante devem participar ativamente de todo o processo. É imprescindível que a equipe multidisciplinar oriente e adote também essas metas, que poderão ajudar a prevenir situações adversas.

As estratégias apresentadas validam que as intervenções e o constante esforço educativo envolvendo pacientes e familiares contribuem para a construção de um cuidado seguro. Reforçam, também, a necessidade de ampliar a discussão do tema e estudos na área.

Dentre os artigos estudados, pode-se perceber que ainda é incipiente a divulgação sobre o cuidado seguro aos pacientes. Nesse sentido, muito ainda deve ser estudado e analisado com relação à adoção de estratégias que permitam maior integração entre profissionais, pacientes e familiares, valorizando-se cada vez mais a atenção à saúde.

\section{REFERÊNCIAS}

AN, Jinok. et al. The effects of patient education on patient safety: can we change patient perceptions and attitudes?: Lessons from the Armed Forces Capital Hospital in Korea. International Journal for Quality in Health care. v. 29, n. 3, p 392-398, jun. 2017. Disponível em: <https://academic.oup.com/intqhc/article/29/3/392/3091654>. Acesso em: 05 maio. 2019.

ARRUDA, Nara Lilia Oliveira. et al. Percepção do Paciente com a segurança no Atendimento em Unidade de Urgência e Emergência. Revista de enfermagem UFPE on line. Recife, v. 11, n. 11, p. 4445-54, nov. 2017. Disponível em: $<$ https://periodicos.ufpe.br/revistas/revistaenfermagem/article/download/15019/24719+\&cd=2\&h l=pt-BR\&ct=clnk\&gl=br>. Acesso em: 5 maio. 2019.

BANDEIRA, Larissa Edom. et al. Condutas de Educação ao Familiar para Promoção da Segurança da Criança Hospitalizada: Registros da Equipe Multiprofissiponal. REME. Revista Mineira de Enfermagem. 2017. Acesso em: 5 maio. 2019. 
BEZERRA, Ana Lúcia Queiroz. et al. Conhecimentos de Usuários de uma Clínica Cirúrgica sobre a Ocorrência de Incidentes. Cogitare Enfermagem. v. 21, n. esp, p. 01-09. 2016. Disponível em: <http://revistas.ufrp.br/cogitare>. Acesso em: 15 out. 2018.

BIASIBETTI, Cecilia. et al. Comunicação para a segurança do paciente em internações pediátricas. Revista Gaúcha Enfermagem, 2019; 40(esp):e20180337. Disponível em:https://doi.org/10.1590/1983-1447.2019.20180337. Acesso em: 11 jun 2020.

BITTENCOURT, Vivian Lemes Lobo. et al. Factors associated with the risk of falls in hospitalized adult patients. Revista Escola de Enfermagem USP. São Paulo, v. 51, e03237, 2017. Disponível em: <http://www.scielo.br/scielo.php?script=sci_arttext\&pid=S008062342017000100435>. Acesso em: 15 fev. 2020.

BRASIL. Portaria no 529, de 1ำ de abril de 2013. Institui o Programa Nacional de Segurança do Paciente (PNSP). Brasília/DF: Ministério de Estado da Saúde, 2013. Disponível em: <http://bvsms.saude.gov.br/bvs/saudelegis/gm/2013/prt0529_01_04_2013.html>. Acesso em: 15 fev. 2020.

BRASIL. Ministério da Saúde. Documento de referência para o Programa Nacional de Segurança do Paciente. Ministério da Saúde; Fundação Oswaldo Cruz; Agência Nacional de Vigilância Sanitária. Brasília: Ministério da Saúde, 2014. 40 p. Disponível em: <http://bvsms.saude.gov.br/bvs/publicacoes/documento_referencia_programa_nacional_segura nca.pdf>. Acesso em: 15 out. 2018.

BRASIL. Agência Nacional de Vigilância Sanitária. Gestão de Riscos e Investigação de Eventos Adversos Relacionados à Assistência à Saúde. Brasília: Anvisa, 2017a. Disponível em:

http://portal.anvisa.gov.br/documents/33852/3507912/Caderno+7++Gest\%C3\%A3o+de+Riscos $+\mathrm{e}+$ Investiga\%C3\%A7\%C3\%A30+de+Eventos+Adversos+Relacionados+\%C3\%A0+Assist\%C3 $\% A A n c i a+\% C 3 \% A 0+S a \% C 3 \% B A d e / 6 f a 4 f a 91-c 652-4 b 8 b-b 56 e-f e 466616 b d 57$. Acesso em 28 de maio 2020.

BRASIL. Agência Nacional de Vigilância Sanitária. Assistência Segura: Uma Reflexão Teórica Aplicada a Prática. Brasília: Anvisa, 2017b. Disponível em: http://portal.anvisa.gov.br/documents/33852/3507912/Caderno+1+-

+ Assist\%C3\%AAncia+Segura+-

+Uma+Reflex\%C3\%A3o+Te\%C3\%B3rica+Aplicada+\%C3\%A0+Pr\%C3\%A1tica/97881798-

cea0-4974-9d9b-077528ea1573. Acessado em: 05 maio 2019.

COSTA, Caroline Lima de Almeida; BORCHARDT, Miriam; PEREIRA, Giancarlo Medeiros. O Papel do Paciente como Coprodutor nos Serviços de Saúde: Uma Revisão Sistemática da Literatura. INTERCIENCIA. v. 43, n. 9, set. 2018. Disponível em: <https://www.interciencia.net/wp-content/uploads/2018/09/648-COSTA-43-09.pdf>. Acesso em: 06 jun. 2019.

FASSARELLA, Cintia Silva. et al. Profissionais Mediadores da Qualidade e Segurança do Paciente como Estratégia para o cuidado seguro. REME. Revista Mineira de Enfermagem. Belo Horizonte, v. 21, e1068, out. 2017. Disponível em: <http://www.reme.org.br/artigo/detalhes/1206>. Acesso em: 3 fev. 2019. 
GOMES, Adriana Pereira Trindade de Souza. et al. Identificação do Paciente em Neonatologia para Assistência Segura. Cogitare Enfermagem. v. 22, n. 3, e49501, jul. 2017. Disponível em: <https://revistas.ufpr.br/cogitare/article/view/49501>. Acesso em: 03 fev. 2019.

LUIZA Melissa de Freitas, et all. Características das quedas com dano em pacientes hospitalizados. Revista Gaúcha Enfermagem. 2019;40. Disponível em: https://www.scielo.br/pdf/rgenf/v40nspe/1983-1447-rgenf-40-spe-e20180307.pdf. Acesso em: 11 jun. 2020

MILAGRES, Lidiane Miranda. Gestão de riscos para segurança do paciente: o enfermeiro e a notificação dos eventos adversos. 2015. 100f. Dissertação (Mestrado em Enfermagem) Universidade Federal de Juiz de Fora, Juiz de Fora, 2015. Disponível em: <http://www.ufjf.br/pgenfermagem/files/2010/05/Disserta\%C3\%A7\%C3\%A3o-Lidiane-MirandaMilagres.pdf>. Acesso em: 03 out. 2017.

PEDRO, Danielli Rafaeli Candido. et al. Conhecimento do paciente sobre a assistência hospitalar recebida durante sua internação. REME. Revista Mineira de Enfermagem. Belo Horizonte, v. 20, e. 978. out. 2016. Disponível em: <http://www.reme.org.br/artigo/detalhes/1114>. Acesso em: 17 jan. 2019.

REBRAENSP. Rede Brasileira de Enfermagem e Segurança do Paciente. Estratégias para a segurança do paciente: manual para profissionais da saúde. Rede Brasileira de Enfermagem e Segurança do Paciente. Porto Alegre: EDIPUCRS, 2013. 132 p. Disponível em: <http://biblioteca.cofen.gov.br/wp-content/uploads/2017/10/Estrat\%C3\%A9gias-paraseguran\%C3\%A7a-do-paciente-manual-para-profissionais-da-sa\%C3\%BAde.pdf>. Acesso em: 15 fev. 2020.

RODRIGUES, Fernanda Araújo. et al. Segurança do Paciente em Unidade Neonatal: Preocupações e Estratégias Vivenciadas por Pais. Cogitare Enfermagem. v. 23, n. 1, e52166, 2018. Disponível em: <https://revistas.ufpr.br/cogitare/article/view/52166>. Acesso em: 6 maio. 2019.

SALVADOR, Pétula Tuani Candido de Oliveira. et al. Análise de Vídeos do Youtube sobre Eventos Adversos em Saúde. REME. Revista Mineira de Enfermagem. v. 18, n. 4, p. 830-837, out./dez. 2014. Disponível em: <http://www.reme.org.br/artigo/detalhes/966>. Acesso em: 17 out. 2018.

SILVA, Thaynara de Oliveira. et al. O envolvimento do paciente na segurança do cuidado: revisão integrativa. Revista Eletrônica de Enfermagem. [Internet]. 2016. Disponível em: <http://docs.bvsalud.org/biblioref/2017/04/832727/33340-183243-1-pb.pdf>. Acesso em: 17 out. 2018.

SOUZA, Ruth Francisca Freitas; SILVA, Lolita Dopico. Estudo exploratório das iniciativas acerca da segurança do paciente em hospitais do Rio de Janeiro. Revista Enfermagem UERJ, Rio de Janeiro, v. 22, n. 1, p. 22-28, jan./fev. 2014. Disponível em: <https://www.epublicacoes.uerj.br/index.php/enfermagemuerj/article/view/11399>. Acesso em: 17 out. 2018.

THORNTON, Kevin C. et al. Prevenção de danos na UTI-Criação de uma cultura de segurança e envolvimento de pacientes e suas famílias. Critical Care Medicine. v. 45, n. 9, p. 1531-1537, set. 2017. Disponível em: <https://insights.ovid.com/crossref?an=00003246-20170900000014>. Acesso em: 17 out. 2018. 
WEGNER, William; PEDRO, Eva Neri Rubim. A segurança do paciente nas circunstâncias do cuidado: prevenção de adventos adversos na hospitalização infantil. Revista LatinoAmericana de Enfermagem. v. 20, n. 3, maio/jun. 2012. Disponível em: <www.eerp.usp.br/rlae>. Acesso em: 17 out. 2018

WORLD HEALTH ORGANIZATION (WHO). World Alliance for Patient Safety. Forward Programme 2008-2009. Geneva: World Health Organization, 2008.

YAN, Jia. et al. Patient reporting of undesirable events: a pilot study in China. Int J Qual Health Care. v. 29, n. 3, p. 360-365, jun. 2017. Disponível em: <https://www.ncbi.nlm.nih.gov/pubmed/28340017>. Acesso em: 21 jun. 2019 